\title{
A Sperner-Type Theorem for Set-Partition Systems
}

\author{
Karen Meagher \\ Department of Mathematics and Statistics \\ University of Ottawa, Ottawa, Ontario, Canada \\ kmeagher@site.uottawa.ca \\ Lucia Moura \\ School of Information Technology and Engineering \\ University of Ottawa, Ottawa, Ontario, Canada \\ lucia@site.uottawa.ca \\ Brett Stevens \\ School of Mathematics and Statistics \\ Carleton University, Ottawa, Ontario, Canada \\ brett@math.carleton.ca
}

Submitted: Aug 15, 2005; Accepted: Oct 26, 2005; Published: Oct 31, 2005

Mathematics Subject Classifications: 05 D05

\begin{abstract}
A Sperner partition system is a system of set partitions such that any two set partitions $P$ and $Q$ in the system have the property that for all classes $A$ of $P$ and all classes $B$ of $Q, A \nsubseteq B$ and $B \nsubseteq A$. A $k$-partition is a set partition with $k$ classes and a $k$-partition is said to be uniform if every class has the same cardinality $c=n / k$. In this paper, we prove a higher order generalization of Sperner's Theorem. In particular, we show that if $k$ divides $n$ the largest Sperner $k$-partition system on an $n$-set has cardinality $\left(\begin{array}{c}n-1 \\ n / k-1\end{array}\right)$ and is a uniform partition system. We give a bound on the cardinality of a Sperner $k$-partition system of an $n$-set for any $k$ and $n$.
\end{abstract}

\section{Introduction}

In this paper, we prove a Sperner-type theorem for systems of set partitions and related results. These theorems are stated after some notation and background results are introduced.

For $i, j$ positive integers with $i \leq j$, let $[i, j]$ denote the set $\{i, i+1, \ldots, j\}$. For $k, n$ positive integers, set $\left(\begin{array}{c}{[n]} \\ k\end{array}\right)=\{A \subseteq[1, n]:|A|=k\}$. This set is also known as a complete $k$-uniform hypergraph. A system $\mathcal{A}$ of subsets of $[1, n]$ is said to be $k$-uniform if $\mathcal{A} \subseteq\left(\begin{array}{c}{[n]} \\ k\end{array}\right)$. 
Two subsets $A, B$ are incomparable if $A \nsubseteq B$ and $B \nsubseteq A$. A set system on an $n$-set $\mathcal{A}$ is said to be a Sperner set system, if any two distinct sets in $\mathcal{A}$ are incomparable.

Sperner's Theorem is concerned with the maximal cardinality of Sperner set systems as well as with the structure of such maximal systems.

Theorem (Sperner's Theorem [14]). A Sperner set system $\mathcal{A}$ of subsets of $[1, n]$ consists of at most $\left(\begin{array}{c}n \\ \lfloor n / 2\rfloor\end{array}\right)$ sets. Moreover, a Sperner set system meets this bound if and only if $\mathcal{A}=\left(\begin{array}{c}{[n]} \\ \lfloor n / 2\rfloor\end{array}\right)$ or $\mathcal{A}=\left(\begin{array}{c}{[n]} \\ \lceil n / 2\rceil\end{array}\right)$.

A sharper version of Sperner's theorem is the LYM Inequality named after Lubell [11], Meshalkin [13] and Yamamoto [15], who each independently established the result.

Theorem (LYM Inequality). Let $n$ be a positive integer and $\mathcal{A}$ be a Sperner set system on an $n$-set. Let $p_{i}$ denote the number of subsets in $\mathcal{A}$ of size $i$, then

$$
\sum_{i=1}^{n} \frac{p_{i}}{\left(\begin{array}{c}
n \\
i
\end{array}\right)} \leq 1 .
$$

A set partition of $[1, n]$ is a set of disjoint non-empty subsets (called classes) of $[1, n]$ whose union is $[1, n]$. Throughout this paper, we refer to set partitions as simply partitions. A partition $P$ is called a $k$-partition if it contains $k$ classes, that is $|P|=k$. Denote by $\mathcal{P}_{k}^{n}$ the set of all $k$-partitions of $[1, n]$. If $n=c k$, a partition $P \in \mathcal{P}_{k}^{n}$ is said to be $c$-uniform if every class of $P$ has the same cardinality, that is $|A|=c$, for all $A \in P$. If $k$ does not divide $n$, a partition $P \in \mathcal{P}_{k}^{n}$ is said to be almost uniform if every class $A \in P$ has $|A|=\lfloor n / k\rfloor$ or $|A|=\lceil n / k\rceil$.

A partition system $\mathcal{P} \subseteq \mathcal{P}_{k}^{n}$ is a Sperner partition system if all distinct $P, Q \in \mathcal{P}$, with $P=\left\{P_{1}, \ldots, P_{k}\right\}$ and $Q=\left\{Q_{1}, \ldots, Q_{k}\right\}$, the following holds

$$
P_{i} \nsubseteq Q_{j} \text {, and } Q_{i} \nsubseteq P_{j} \text {, for all } i, j \in\{1, \ldots, k\} \text {. }
$$

Let $\operatorname{SP}(n, k)$ denote the size of the largest Sperner partition system in $\mathcal{P}_{k}^{n}$.

If $\mathcal{P}$ is a partition system, then $\mathcal{P}$ is a Sperner partition system if and only if all the partitions in $\mathcal{P}$ are disjoint (no two partitions have a common class) and the union of all the classes of all the partitions in $\mathcal{P}$ is a Sperner set system. In design theory, a collection of disjoint subsets of an $n$-set whose union is the $n$-set is called a resolution class. Any set system that can be partitioned into resolution classes is called resolvable. In this sense, Sperner partition systems can be considered resolvable Sperner set systems.

There have been extensions of Sperner's Theorem to systems of families of sets [5] and to systems of subsets of a set $X$ with a 2-partition $X=X_{1} \cup X_{2}$ such that no two subsets $A, B$ in the system satisfy both $A \cap X_{i}=B \cap X_{i}$ and $A \cap \overline{X_{i}} \subseteq B \cap \overline{X_{i}}$ where $i \in\{1,2\}[6,8,9]$. Our notion of a Sperner partition system is quite different; our result extends Sperner's Theorem from sets to set-partitions. A related extension of the Erdős-Ko-Rado Theorem to set partitions is found in [12].

Bollobás [2] gives a generalization of the LYM Inequality to two families of sets. For positive integers $n, m$ let $\mathcal{A}=\left\{A_{i}, B_{i}: i=1, \ldots, m\right\}$ be a set system of subsets from $[1, n]$ 
with the property that $A_{i} \cap B_{i} \neq \emptyset$ and $A_{i} \not A_{j} \cup B_{j}$ for $i \neq j$. Then $\sum_{i=1}^{m}\left(\begin{array}{c}n-\left|B_{i}\right| \\ \left|A_{i}\right|\end{array}\right) \leq$ 1. This result implies both Sperner's Theorem and the LYM Inequality but does not generalize to three families of sets.

Another generalization of Sperner's Theorem that involves partition systems looks at the poset of partitions ordered by refinement $[3,4]$. This has no direct relationship to our result as a system of $k$-partitions can not contain both a partition and any of its refinements.

Our first result is the exact size of the largest Sperner partition system in $\mathcal{P}_{k}^{n}$ when $k$ divides $n$.

Theorem 1. Let $n, k, c$ be integers with $n=k c$. Then $\operatorname{SP}(n, k)=\left(\begin{array}{c}c k-1 \\ c-1\end{array}\right)$. Moreover, $a$ Sperner partition system meets this bound only if it is a c-uniform partition system.

Our second result is a bound on the size of $\mathrm{SP}(n, k)$ for general $n$ and $k$.

Theorem 2. Let $n, k, c$ and $r$ be integers with $n=c k+r$ and $0 \leq r<k$. Then

$$
\mathrm{SP}(n, k) \leq \frac{1}{(k-r)+\frac{r(c+1)}{n-c}}\left(\begin{array}{l}
n \\
c
\end{array}\right) .
$$

In Section 2, we prove the bound on the size of a Sperner partition system stated in Theorem 2. In Section 3, we prove Theorem 1.

\section{A Bound on the Cardinality of Sperner Partition Systems in $\mathcal{P}_{k}^{n}$}

Theorem 2 states that for integers $n, k, c, r$ with $n=c k+r$ and $0 \leq r<k$, we have the following bound on the cardinality of a Sperner partition system in $\mathcal{P}_{k}^{n}$ :

$$
\mathrm{SP}(n, k) \leq \frac{\left(\begin{array}{l}
n \\
c
\end{array}\right)}{(k-r)+\frac{r(c+1)}{n-c}} .
$$

Proof of Theorem 2. If $k=1$ then $n=c$ and $r=0$. Further, $\mathcal{P}_{k}^{n}$ has only one partition, namely $\{\{1, \ldots, n\}\}$ and the theorem holds trivially. So we assume that $k \geq 2$.

Let $\mathcal{P} \subseteq \mathcal{P}_{k}^{n}$ be a Sperner partition system. Let $\mathcal{A}$ be the Sperner set system formed by taking all classes from all the partitions in $\mathcal{P}$. Thus $|\mathcal{A}|=k|\mathcal{P}|$.

Let $p_{i}, i \in\{1, \ldots, n\}$ be the number of sets in $\mathcal{A}$ with size $i$. By the LYM Inequality

$$
\sum_{i=1}^{n} \frac{p_{i}}{\left(\begin{array}{l}
n \\
i
\end{array}\right)} \leq 1
$$

Following the notation from [7], define the function $f(i)=\left(\begin{array}{c}n \\ i\end{array}\right)^{-1}$. With this, we get

$$
\sum_{i=1}^{n} \frac{p_{i}}{|\mathcal{A}|} f(i) \leq \frac{1}{|\mathcal{A}|}
$$


In [7], it is shown that the function $f(i)$, for $i=1, \ldots, n$, can be extended to a convex function on the real numbers by

$$
f(i+u)=(1-u) f(i)+u f(i+1), \text { for } 0 \leq u \leq 1
$$

Since the set system $\mathcal{A}$ is formed from a $k$-partition system on an $n$-set,

$$
\sum_{i=1}^{n} i p_{i}=\sum_{A \in \mathcal{A}}|A|=n|\mathcal{P}|=n \frac{|\mathcal{A}|}{k} .
$$

Using the above equality, the fact that $f(i)$ is a convex function with $\sum_{i=1}^{n} \frac{p_{i}}{|\mathcal{A}|}=1$, and Equation (2), we get

$$
f\left(\frac{n}{k}\right)=f\left(\sum_{i=1}^{n} i \frac{p_{i}}{|\mathcal{A}|}\right) \leq \sum_{i=1}^{n} f(i) \frac{p_{i}}{|\mathcal{A}|} \leq \frac{1}{|\mathcal{A}|} .
$$

From the definition of $f(i)$ we get

$$
f\left(\frac{n}{k}\right)=f\left(\frac{c k+r}{k}\right)=f\left(c+\frac{r}{k}\right)=\left(1-\frac{r}{k}\right)\left(\begin{array}{c}
n \\
c
\end{array}\right)^{-1}+\frac{r}{k}\left(\begin{array}{c}
n \\
c+1
\end{array}\right)^{-1} .
$$

Thus,

$$
|\mathcal{A}| \leq \frac{1}{\left(1-\frac{r}{k}\right)\left(\begin{array}{l}
n \\
c
\end{array}\right)^{-1}+\frac{r}{k}\left(\begin{array}{c}
n \\
c+1
\end{array}\right)^{-1}}=\left(\begin{array}{l}
n \\
c
\end{array}\right) \frac{k}{(k-r)+\frac{r(c+1)}{n-c}}
$$

and

$$
|\mathcal{P}| \leq \frac{\left(\begin{array}{l}
n \\
c
\end{array}\right)}{(k-r)+\frac{r(c+1)}{n-c}}
$$

We would like to know the exact cardinality and structure of the largest Sperner partition system. We conjecture that the largest Sperner partition system is an almostuniform partition system.

Conjecture. Let $n, k$ be positive integers. The largest Sperner partition system in $\mathcal{P}_{k}^{n}$ is an almost-uniform partition system.

For the case where $n=c k$, where $c$ and $k$ are integers, an almost-uniform partition system is a uniform partition system. In the next section, we prove this conjecture for this case. Specifically, we show that the largest Sperner partition system in $\mathcal{P}_{k}^{c k}$ is a uniform partition system. 


\section{Sperner's Theorem for Partition Systems in $\mathcal{P}_{k}^{c k}$}

When $n=c k$, a 1-factor of the complete uniform hypergraph $\left(\begin{array}{c}{[n]} \\ c\end{array}\right)$ is equivalent to a uniform $k$-partition of an $n$-set, and a 1-factorization of $\left(\begin{array}{c}{[n]} \\ c\end{array}\right)$ corresponds to a Sperner partition system. If $c$ divides $n$, the hypergraph $\left(\begin{array}{c}{[n]} \\ c\end{array}\right)$ has a 1-factorization with $\left(\begin{array}{l}n-1 \\ c-1\end{array}\right)$ factors. Rephrasing this result using the above equivalence, we get the following result.

Theorem (Baranyai [1]). Let $n, k, c$ be positive integers with $n=c k$, then there exists a Sperner partition system in $\mathcal{P}_{k}^{n}$ of cardinality $\left(\begin{array}{l}n-1 \\ c-1\end{array}\right)$.

The proof that this is the largest Sperner partition system uses a result by Kleitman and Milner on Sperner set systems. For a set system $\mathcal{A}$, define the volume of $\mathcal{A}$ to be $t(\mathcal{A})=\sum_{A \in \mathcal{A}}|A|$.

Theorem (Kleitman and Milner [10]). Let $\mathcal{A}$ be a Sperner set system on an $n$-set, with $|\mathcal{A}| \geq\left(\begin{array}{l}n \\ c\end{array}\right)$ and $c \leq \frac{n}{2}$, then

$$
\frac{t(\mathcal{A})}{|\mathcal{A}|} \geq c
$$

This inequality is strict in all cases except when $\mathcal{A}=\left(\begin{array}{c}{[n]} \\ c\end{array}\right)$.

Proof of Theorem 1. If $k=1$ then $\mathcal{P}_{k}^{n}$ has only one partition, namely $\{\{1, \ldots, n\}\}$, and the theorem holds trivially. So we assume that $k \geq 2$.

Let $n, k$ and $c$ be positive integers with $n=k c$. From Theorem 2, with $r=0$ and Baranyai's Theorem, $\mathrm{SP}(n, k)=\frac{1}{k}\left(\begin{array}{l}n \\ c\end{array}\right)$.

It remains to show that if a Sperner partition system meets this bound then it is $c$-uniform. Assume that $|\mathcal{P}|=\frac{1}{k}\left(\begin{array}{l}n \\ c\end{array}\right)$. Let $\mathcal{A}$ be the Sperner set system formed by taking all classes from all the partitions in $\mathcal{P}$, then $|\mathcal{A}|=\left(\begin{array}{l}n \\ c\end{array}\right)$ and $c \leq \frac{n}{2}$.

Since $\frac{t(\mathcal{A})}{|\mathcal{A}|}=\frac{\Sigma_{A \in \mathcal{A}}|A|}{|\mathcal{A}|}=c$, from Kleitman and Milner's theorem, it follows that $\mathcal{A}=\left(\begin{array}{c}{[n]} \\ c\end{array}\right)$ and $\mathcal{P}$ is a uniform partition system.

Theorem 1 is a natural extension of Sperner's Theorem. Sperner's Theorem says that the Sperner set system with maximum cardinality on an $n$-set is the collection of all $\left\lfloor\frac{n}{2}\right\rfloor$ sets. Theorem 1 says that for integers $n, k$ such that $k$ divides $n$, the Sperner $k$-partition system on an $n$-set with the largest cardinality is the collection of all $\left(\frac{n}{k}\right)$-sets arranged in resolution classes.

The following corollary follows from Theorem 1 and the fact that $\operatorname{SP}(n, k)$ is a nondecreasing function for fixed $k$.

Corollary 3. Let $n, k, c$ be positive integers with $n=c k+r$ where $0 \leq r<k$. Then

$$
\frac{1}{k}\left(\begin{array}{c}
c k \\
c
\end{array}\right) \leq \mathrm{SP}(n, k) \leq \frac{1}{k}\left(\begin{array}{c}
(c+1) k \\
c+1
\end{array}\right) \text {. }
$$

From Corollary 3, we can calculate the asymptotic growth of $\operatorname{SP}(n, k)$ for the general case:

$$
\lim _{n \rightarrow \infty} \frac{\log \operatorname{SP}(n, k)}{n}=\log k-\frac{k-1}{k} \log (k-1) .
$$




\section{References}

[1] Z. Baranyai. On the factorization of the complete uniform hypergraph. In Infinite and finite sets (Colloq., Keszthely, 1973; dedicated to P. Erdös on his 60th birthday), Vol. I, pages 91-108. Colloq. Math. Soc. Jánōs Bolyai, Vol. 10. North-Holland, Amsterdam, 1975.

[2] B. Bollobás. On generalized graphs. Acta Math. Acad. Sci. Hungar., 16:447-452, 1965.

[3] E. R. Canfield. On a problem of Rota. Bull. Amer. Math. Soc., 84(1):164, 1978.

[4] E. R. Canfield. On a problem of Rota. Adv. in Math., 29(1):1-10, 1978.

[5] D. E. Daykin, P. Frankl, C. Greene, and A. J. W. Hilton. A generalization of Sperner's theorem. J. Austral. Math. Soc. Ser. A, 31(4):481-485, 1981.

[6] P. L. Erdős and G. O. H. Katona. A 3-part Sperner theorem. Studia Sci. Math. Hungar., 22(1-4):383-393, 1987.

[7] M. Frédéric. On the flat antichain conjecture. Australas. J. Combin., 15:241-245, 1997.

[8] G. Katona. On a conjecture of Erdős and a stronger form of Sperner's theorem. Studia Sci. Math. Hungar., 1:59-63, 1966.

[9] D. J. Kleitman. On a lemma of Littlewood and Offord on the distribution of certain sums. Math. Z., 90:251-259, 1965.

[10] D. J. Kleitman and E. C. Milner. On the average size of the sets in a Sperner family. Discrete Math., 6:141-147, 1973.

[11] D. Lubell. A short proof of Sperner's lemma. J. Combinatorial Theory, 1:299, 1966.

[12] K. Meagher and L. Moura. Erdős-Ko-Rado theorems for uniform set-partition systems. Electron. J. Combin., 12(1):Research Paper 40, 12 pp. (electronic), 2005.

[13] L. D. Mešalkin. A generalization of Sperner's theorem on the number of subsets of a finite set. Teor. Verojatnost. i Primenen, 8:219-220, 1963.

[14] E. Sperner. Ein Satz über Untermengen einer endlichen Menge. Math. Z., 27:544-548, 1928.

[15] K. Yamamoto. Logarithmic order of free distributive lattice. J. Math. Soc. Japan, 6:343-353, 1954. 